"This is an original manuscript of the article published by: S. Laureti, P. Burrascano, H. Malekmohammadi, M. Ricci, S. Sfarra, in '5th IEEE International Workshop on Metrology for AeroSpace, Rome, Italy. June 2018.

Available online at: https://ieeexplore.ieee.org/document/8453581 DOI: 10.1109/MetroAeroSpace.2018.8453581

"(C) 2018 IEEE. Personal use of this material is permitted. Permission from IEEE must be obtained for all other uses, in any current or future media, including reprinting/republishing this material for advertising or promotional purposes, creating new collective works, for resale or redistribution to servers or lists, or reuse of any copyrighted component of this work in other works." 


\section{Auto Regressive Exogenous modeling of pseudo- noise thermography for the nondestructive evaluation of aerospace materials}

\author{
S. Laureti ${ }^{1}$, P. Burrascano ${ }^{1}$, H. Malekhommadi ${ }^{1}$ \\ Dept. of Engineering, University of Perugia \\ Polo scientifico didattico di Terni, Strada di Pentima 4, \\ 05100, Terni, ITALY \\ \{stefano.laureti, pietro.burrascano, \\ hamed.malekmohammadi\}@unipg.it
}

\begin{abstract}
An Auto Regressive Exogenous model is proposed for the feature extraction in Random Photothermal active Thermography. The prosed approach is applied to the nondestructive evaluation of a Carbon Fiber Reinforced Plastic composite sample in which delamination was simulated by embedding Teflon tape pieces between the different plies of the structure. It is shown that the application of the Auto Regressive Exogenous model allows the impulse response of the sample to be estimated, thus simulating the result of a standard PulsedThermography experiment but by using a pseudo-noise modulated low-power heating source. Such technique opens the possibility to realize handier setup and to achieve higher SNR values than Pulsed-Thermography. For these reasons the proposed approach can find use in the nondestructive evaluation of aerospace composite components.
\end{abstract}

Keywords-nondestrucitve evaluation; thermography, autoregressive exogenous model, composite, aerospace, pseudo-noise;

\section{INTRODUCTION}

Active Thermography (AT) is a Nondestructive Testing (NDT) technique extensively used in various fields of research and analysis, such as material characterization, online and off-line industrial products quality control and cultural heritage diagnostic [1]. In AT, the needed thermal contrast is obtained by exciting the Sample Under Test (SUT) with an external heating stimulus. Several different AT schemes have been developed, which depend on the time characteristics of the adopted heating stimulus. Pulsed Thermography (PT) is one of the most employed AT schemes. In PT, the SUT is excited by a short heating stimulus often provided by a flash-head lamp, which can be considered a good approximation of the Dirac's Delta $\delta(t)$. Therefore, the acquired InfraRed (IR) response represents the pixelwise impulse response $h(t)$ of the SUT. The features of interest are retrieved by performing a time analysis of both the heating and cooling trends of the $h(t)$. Although PT is relatively easy to be implemented, and a high number of features can be extracted from the acquired data due to the broadband nature of the input $\delta(t)$, high-power heating sources, i.e. high energy flashes, must be used to guarantee an adequate thermal contrast allowing inner defects to be detected. Two major drawbacks can therefore arise: (1) the pulsed heating stimulus

This research work has been partially supported from the European Union's Horizon 2020 research and innovation programme under the Marie Skłodowska-Curie grant agreement No 722134 - NDTonAIR

\author{
M. Ricci ${ }^{2}$, S. Sfarra ${ }^{3}$ \\ ${ }^{2}$ DIMES, University of Calabria, Via Pietro Bucci, 87036 \\ Arcavacata, Rende CS, ITALY \\ ${ }^{3}$ DIIEE, University of L'Aquila, Piazzale E. Pontieri 1, \\ 67100 Loc. Monteluco di Roio - L'Aquila (AQ), ITALY \\ m.ricci@dimes.unical.it; stefano.sfarra@univaq.it
}

may provoke abrupt variations of the sample surface temperature, potentially affecting the chemical properties of the inspected item, (2) the experimental set-up can be expansive and cumbersome. A possible strategy to tackle both these issues is that of spreading the heating stimulus energy over a longer time interval, allowing hence the use of lowpower heating sources. This can be done by heating up the SUT with either a constant heat flux, i.e. Step-Heating Thermography (SHT), or an amplitude-modulated heat flux, as in Lock-in Thermography (LT). However, measurement performed by SHT suffers from low SNR at high frequencies. On the other hand, LT requires an a priori knowledge of the SUT thermal properties to select a suitable modulation frequency, though providing a significant gain in the SNR. In the last two decades, efforts have been made to combine the effectiveness and simplicity of PT and the SNR gain provided by LT, leading for example to Pulsed-Phase Thermography, or Multi-Frequency Lock-in Thermography [2]. To this end, the use of coded heating stimulus in the form of either a frequency modulated "chirp" signal or of a pseudo-noise sequence showing a delta-like auto-correlation function it has been also proposed. The use of such coded heating stimulus allows a frequency analysis to be directly performed on the acquired raw data, whilst a time analysis can be carried out once the $h(t)$ is retrieved. Two main approaches have been used for this purpose: (i) a deterministic cross-correlation based algorithm known as Pulse-Compression, i.e. PulseCompression Thermography (PuCT), which consists on the application of the so-called Matched Filter over the pixelwise acquired IR data [3-9]; (ii) the use of an Auto Regressive Moving Average (ARMA) model, together with a Least Square Minimization algorithm for estimating the systems transfer function, thus retrieving the $h(t)$. Approach (ii) is referred to Random Photothermal Thermography (RPT) [10]. Both the said approaches have shown promises for extracting the features of interest from IR data. For instance, the achieved SNR gain in the estimated $h(t)$ with respect to PT is proportional to the time duration of the coded signal. In case a high sensitivity should be requested, the inspection capability can be then tuned at the cost of increasing the measurement time. However, a detailed guideline on how to implement the method (ii) in RPT is missing, as well as a detailed time domain analysis of the retrieved $h(t)$. 
In this paper, the use of an Auto-Regressive eXogenous model (ARX) to both estimating the system's $h(t)$ and to extract features in RPT is proposed. Although other approaches based on neural networks and machine learning algorithms might have been here employed for feature extraction purposes, the ARX model represents a robust choice for an accurate system's $h(t)$ identification $[11,12]$. The quality of the investigated ARX approach is shown in detail. To this aim, a Maximum Length Sequence (MLS) pseudo-noise signal was used to modulate the heat source emission, consisting here of eight LED chips. The benchmark sample was here a Carbon Fiber Reinforced Plastic (CFRP) slab containing nine thin artificial flaws embedded at different depths. An SNR quantitative time analysis of the feature extraction capability for the proposed method is given. Finally, it is shown that the employed system's identification algorithm lead to a faithful reconstruction of the impulse response, allowing advanced post-processing algorithms to be successfully employed. In fact, the so-called "Time-Phase" algorithm [5] has been here exploited to enhance the detection capability of defects at deeper depth within the SUT. Note that although Time-Phase algorithm has been fruitfully applied for both Pseudorandom-Noise Photoacoustic and PuCT setups [5,8,9], there is no evidence in literature of its exploitation in RPT (at the best of our knowledge).

\section{SYSTEM'S IDENTIFICATION AND ARX MODEL}

Within the equation error family, the ARX models represent the simplest way to identify the unknown mechanism that generates a data set that generates a data set whose statistical characteristics are those of the desired signal. In this work, an ARX is used to model the unknown IR response of the SUT when excited by a pseudo-noise heating stimulus. To successfully design an ARX model of the system, both the input signal $x(t)$ and the output signal $y(t)$ must be totally or partially available. Input and output data are collected by sampling continuous time signals and the subsequent processing is carried out in discrete time. Eq.(1) shows thus the general input/output relation for an ARMA model in the discrete time domain, normalized to the sampling frequency $f_{\mathrm{s}}$, e.g. $y[k]=\left.y(t)\right|_{t=k / f_{s}}[13]$ :

$$
A[q] y[k]=B[q] x\left[k-n_{k}\right]+C[q] e[k]
$$

In Eq.(1) $n_{k}$ is the input - output delay, i.e. the dead-time of the system, the sequence $e[k]$ is the model output error (residual), i.e. the difference between the model output $y_{\text {mod }}[k]$ and $y[k] ; q$ is the delay operator defined by: $q y[k]=$ $y[k+1]$; the polynomials:

$$
\begin{aligned}
& A[q]=q^{n}+a_{1} q^{n-1}+\cdots+a_{n} \\
& B[q]=b_{1} q^{n-1}+\cdots+b_{n} \\
& C[q]=q^{n}+c_{1} q^{n-1}+\cdots+c_{n}
\end{aligned}
$$

of given orders, $n_{a}, n_{b}$ and $n_{c}$ respectively, allow the synthetic description in Eq.(1) of the differences equation to be given:

$$
\begin{aligned}
y[k]+a_{1} y[k-1]+ & \cdots+a_{n} y[k-n]= \\
& =b_{1} x\left[k-n_{k}-1\right]+\cdots \\
& +b_{n} x\left[k-n_{k}-n\right]+e[k] \\
& +c_{1} e[k-1]+\cdots+c_{n} e[k-n]
\end{aligned}
$$

An ARX model is a simplified version of the general ARMA model as shown in Eq.(3):

$$
y[k]=\frac{B[q]}{A[q]} x\left[k-n_{k}\right]+\frac{1}{A[q]} e[k]
$$

Eq.(3) it is equivalent to Eq.(1) when $n_{c}=0$. In this case, Eq.(3) becomes the extended input/output linear difference equation of the ARX model:

$$
\begin{aligned}
& y[k]+a_{1} y[k-1]+\cdots+a_{n_{a}} y\left[k-n_{a}\right]=b_{1} x\left[k-n_{k}\right]+ \\
& +\cdots+b_{n_{b}} x\left[k-\left(n_{k}+n_{b}-1\right)\right]+\mathrm{e}[k]
\end{aligned}
$$

The problem of the system's modelling is thus strictly related on choosing optimal values for $\left(n_{a}, n_{b}, n_{k}\right)$ and calculating the coefficients $\left(a_{1}, \ldots, a_{n_{a}}\right),\left(b_{1}, \ldots, b_{n_{b}}\right)$ that minimize the residual error $e[k]$. Once the said parameters have been selected, the systems impulse response $h[k]$ can be estimated by imposing $x[k]=\delta[k]$ on the obtained optimal polynomial model.

The best fit parameter values give a good fit of the given data, and thus the power of model residual $e[k]$ is minimized; the residual noise sequence shows both a white-noise like autocorrelation and a quasi-zero amplitude of the crosscorrelation function [13-18].

\section{EXPERIMENTAL SETUP AND METHOD}

A detailed sketch of the experimental setup is depicted in Fig.1.

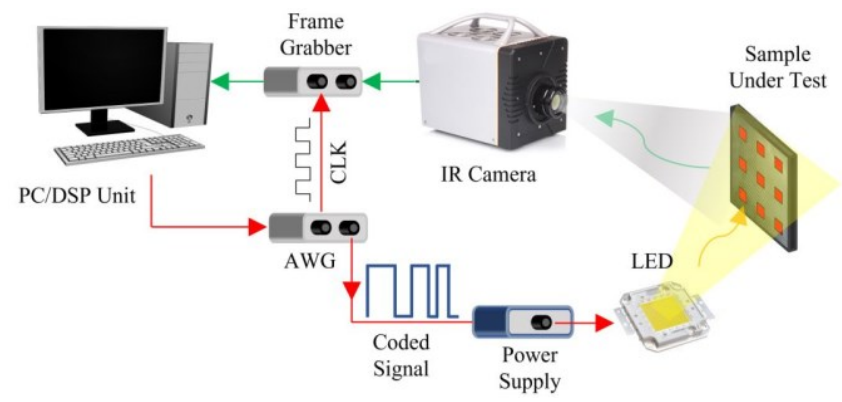

Fig.1. A sketch of the experimental setup.

A PC/DSP unit with an in-house virtual instrument developed in LabVIEW $^{\circledR}$ managed the signal acquisition/generation. A National Instrument PCI-6711 Arbitrary Waveform Generator (AWG) board provided both the MLS excitation and a reference clock signal (CLK) for triggering the Xenics Onca-MWIR-InSb camera acquisition. Note that thermograms were acquired at 40 FPS. A TDK Lambda GEN $750 \mathrm{~W}$ power supply fed eight LED chips placed at about 30 $\mathrm{cm}$ from the SUT with the wanted coded excitation modulation. The LED system provided a maximum power of $400 \mathrm{~W}$. The SUT was a twelve plies $0^{\circ}-90^{\circ}$ CFRP sample, containing nine thin Teflon ${ }^{\circledR}$ defects buried at different depths within the sample. The defects had lateral dimension of $20 \mathrm{x}$ $20 \mathrm{~mm}$ and a thickness of $\sim 75 \mu \mathrm{m}$. A quoted sketch of the SUT is shown in Fig.2, whereas the location of each defect into the sample is depicted as well. The nine defects were 


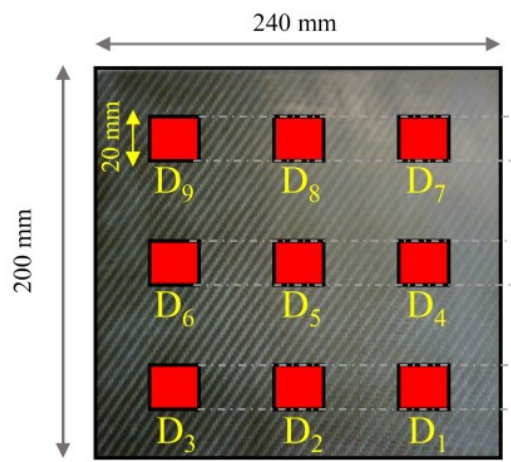

ply thickness $\sim 0.23 \mathrm{~mm}$

Fig.2. A quoted sketch of the sample under test.
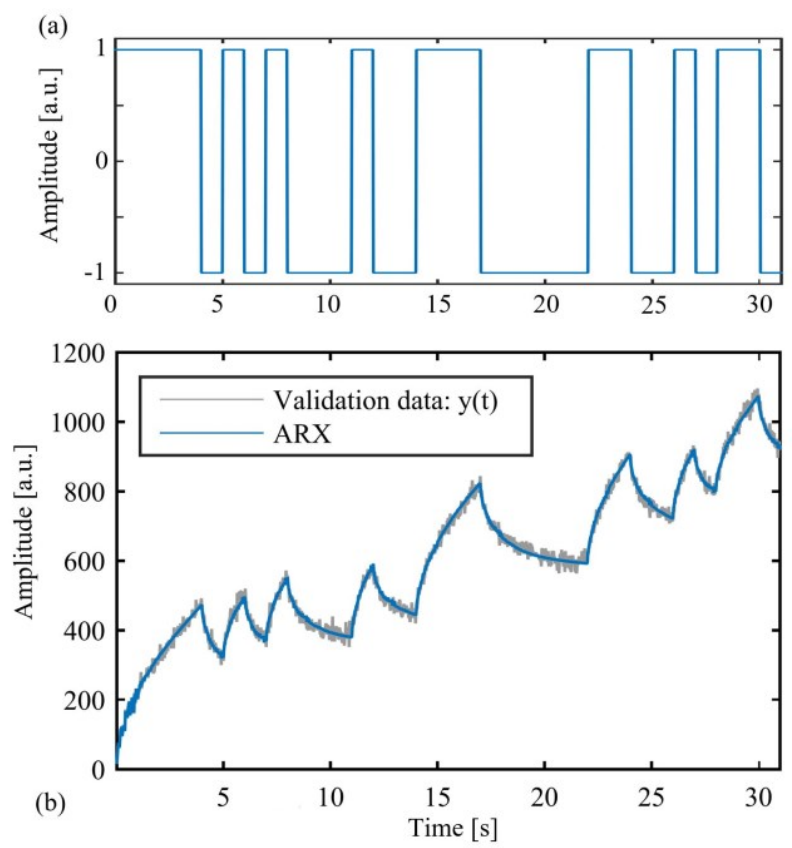

Fig.3. (a) MLS coded input signal. (b) SUT's IR raw acquire signal from a single pixel (gray) and polynomial fit obtained from $\operatorname{ARMA}(40,40,0)$ (blue).
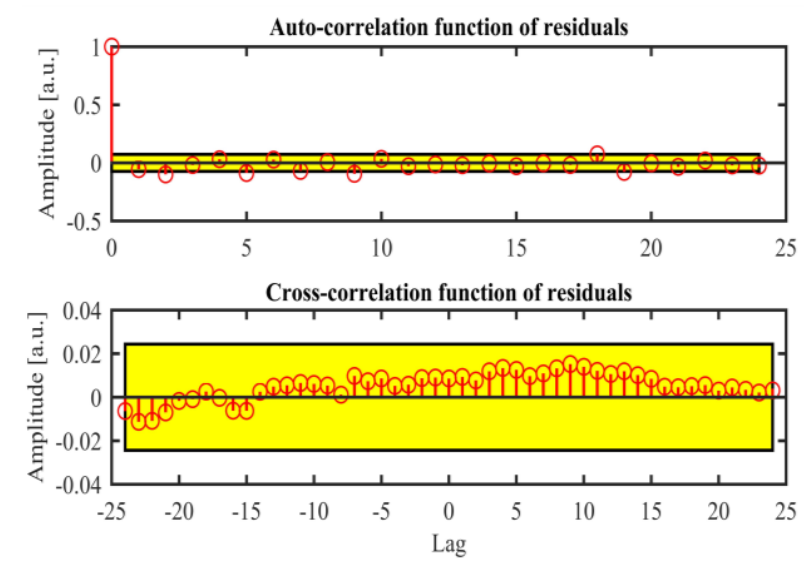

Fig.4. Auto- and Cross- correlation of the residuals $e(t)$. named " $\mathrm{D}_{1}$ " to " $\mathrm{D}_{9}$ ", with higher value of depth from the inspected SUT surface as the subscript value increases.

The coded signal used to modulate the LED emission was a pseudo-noise binary MLS of order $n=5$, resulting in an overall bit duration $p=2^{5}-1$. Each " +1 " or " -1 " of the MLS drove for an "On" and "Off" state of the LED system respectively. The single " +1 " and " -1 " bits of the MLS signal lasts for $T_{s}=1 \mathrm{~s}$ for the chosen generation rate of $40 \mathrm{~S} / \mathrm{s}$, resulting in an overall signal time duration $T$ equal to 31 seconds $[20,21]$. Note, that a $T_{s}=1 \mathrm{~s}$ was chosen to guarantee the onset of thermal waves having relatively high thermal diffusion length. This in turn assures a good diffusion of the thermal waves into the sample. The reader is referred to Laureti et al. for further details on how to design a coded signal for inspecting a specific sample in AT [8]. The designed MLS input signal $x[k]$ is shown in Fig.3(a).

\section{RESULTS}

The gray line plot in Fig.3(b) shows the SUT's acquire raw IR time response $y[k]$ over the impinging MLS-modulated heating stimulus (see Fig.3(a)). The prediction capability of the proposed $\operatorname{ARX}(40,40,0)$ model is depicted with a blue line plot in Fig.3(b), where a good fit with the $y[k]$ is appreciated.

The fit quality is quantitatively evaluated by considering the value of the Normalized Root Mean Square Error (NRMSE) obtained from ARX prediction over the $y[k]$ test, which was equal to $93.34 \%$. Both the auto- and cross-correlation functions of the residual error $e[k]$ have been also considered to further corroborate the chosen model - their handling is depicted in Fig.4. The auto- and cross- correlation functions were plotted against different values of Lag operator (backward shifting operator). The auto-correlation function is in the form of a white-noise one (delta-like) and its amplitude lies within the chosen confidence level here highlighted in yellow, as happens for the cross-correlation amplitude as well. A zoom of the reconstructed impulse response $h[k]$, which have been estimated by fitting the values of the obtained polynomials $A$ and $B$ within a time range of interest, is shown in Fig.5.

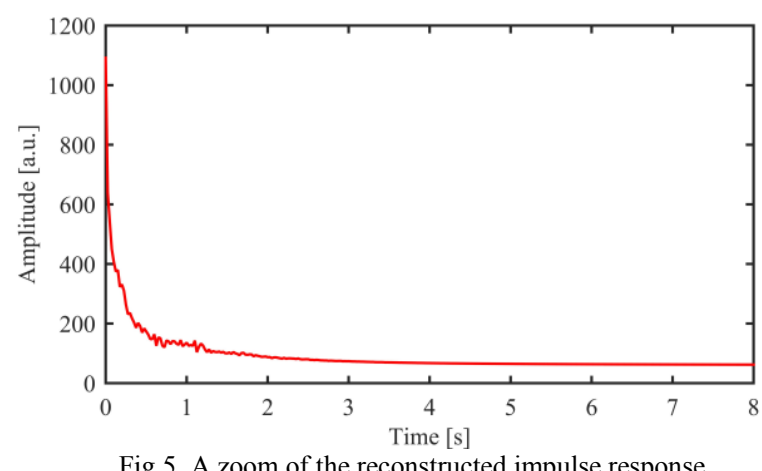

Fig.5. A zoom of the reconstructed impulse response.

The $h[k]$ shows an exponential decay trend, thus in a good agreement with the expected IR response of the SUT (modelled as a linear system) when a pseudo-noise excitation is used to modulate the impinging heat $[6,8]$. 

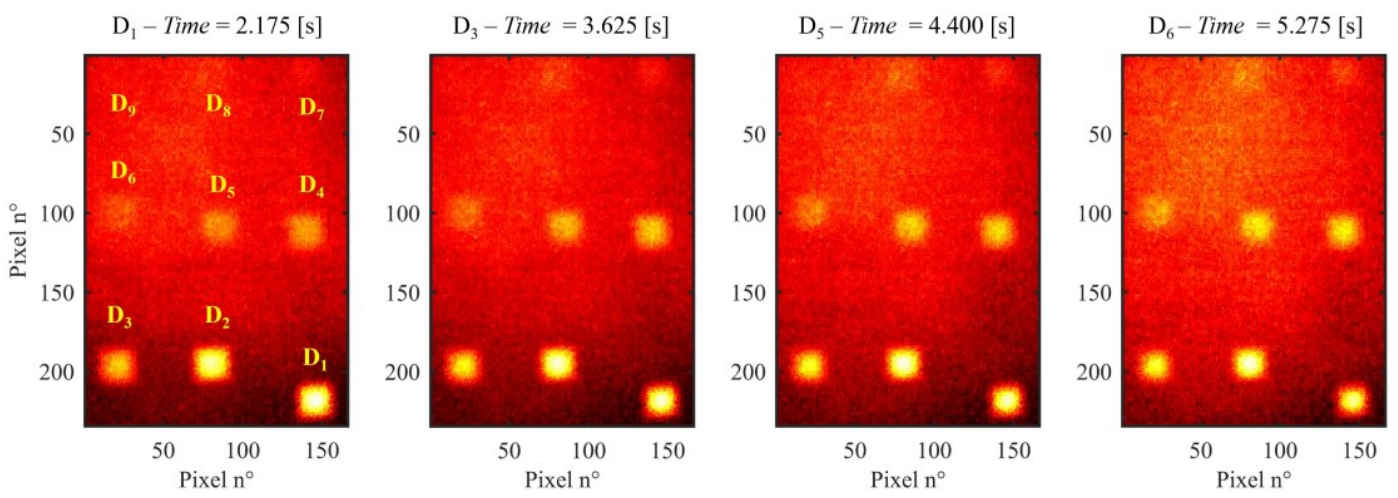

Fig.7. A series of thermograms obtained at the time instant at which $S N R(t)$ reaches its maximum value for $\mathrm{D}_{1}, \mathrm{D}_{3}, \mathrm{D}_{5}, \mathrm{D}_{6}$ respectively. The position of the nine defects has been labelled on the left thermogram.

Consequently, the $h[k]$ has been retrieved for each $\mathrm{x}$-y pixel of the acquired IR thermograms, following a pixelwise reconstruction with the described ARX method. Thus, a merit parameter in the form of $S N R(t)$ was defined to appreciate the feature extraction capability of the proposed model:

$$
S N R_{D_{j}}[k]=\frac{h_{D_{j}}[k]-\bar{h}[k]}{\sigma_{h}[k]}
$$

where $h_{D_{j}}[k]$ is the impulse response of the $j$-th defect $(j=1,2, \ldots, 9)$ averaged over a $4 \times 4$ pixels area onto the defect, $\bar{h}[k]$ is the impulse response averaged over all the SUT area and $\sigma_{h}[k]$ is its standard deviation. Fig.6 shows the obtained $S N R_{D_{j}}[k]$ : defects $\mathrm{D}_{1}-\mathrm{D}_{6}$ are well detected; defects located at deeper depths within the sample, i.e. $\mathrm{D}_{7}$ and $\mathrm{D}_{9}$, show $\mathrm{SNR}$ maxima $<1$, thus they are not detected. Furthermore, $\mathrm{D}_{8}{ }^{\text {c }} \mathrm{s}$ signature is misread as buried in noise. For the well-detected defects $\mathrm{D}_{1}-\mathrm{D}_{6}$, the corresponding $S N R_{D_{j}}[k]$ maxima are reached at different time as they are buried at different depths, thus showing promises for a faithful time analysis.

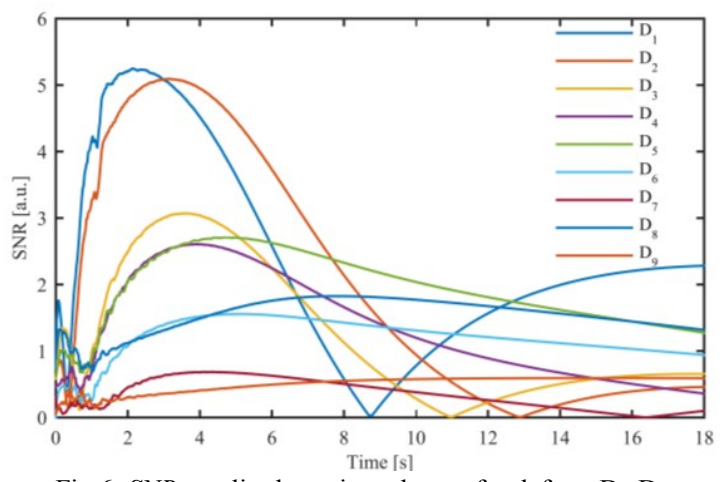

Fig.6. $S N R$ amplitude as time elapses for defects $\mathrm{D}_{1}-\mathrm{D}_{9}$.
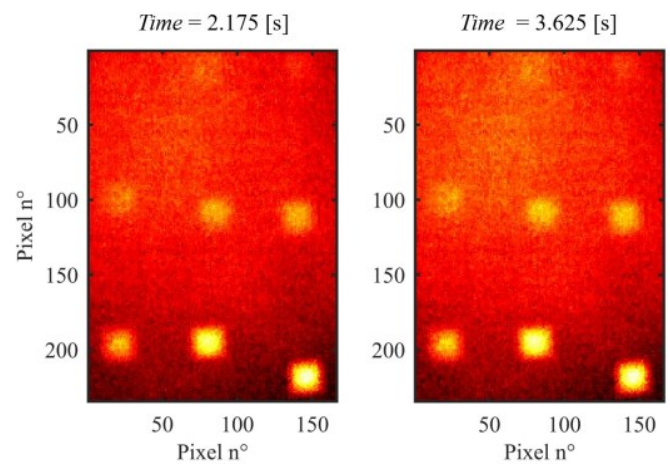

Fig.9. A series of thermograms for the for the $\operatorname{ARX}(2,4,0)$ obtained at the same time instant investigated for $\operatorname{ARX}(40,40,0)$ (see Fig.8).
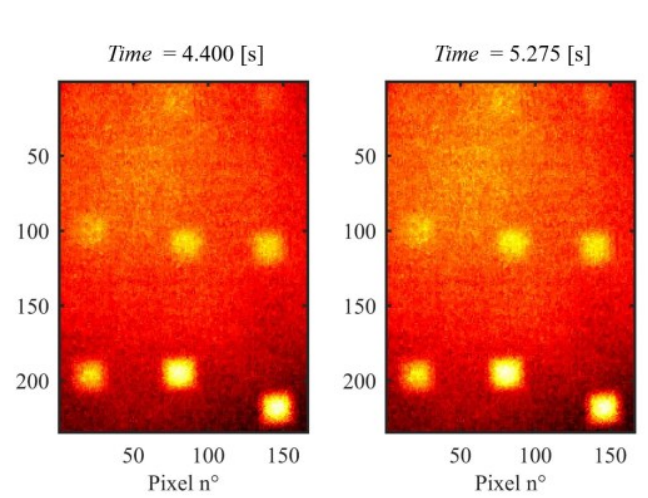

To this aim, Fig.7 shows the thermograms corresponding at

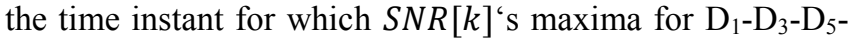
$\mathrm{D}_{6}$ are reached. The investigated defects appear as brighter and brighter pixel areas as time elapses. To further investigate the robustness of the proposed approach for features in RPT, the same analysis was carried out by modelling the system with a lower order ARX model, for which $n_{a}=2, n_{b}=4, n_{k}$ $=0$ were selected. Fig. 8 shows the $S N R_{D_{j}}[k]$ amplitude obtained for the ARX $(2,4,0)$.

It can be noted that while lowering the ARX order still allows a fruitful feature extraction to be carried out (SNR $>1)$, the possibility of performing a time analysis is hampered by the choice of a low order ARX model. In fact, flattened $S N R[k]$ are obtained, as showed in Fig.8. This can be further appreciated in Fig. 9, where thermograms obtained with both ARX $(2,4,0)$ are shown for the same time instants of the one retrieved with ARX $(40,40,0)$. Indeed, a constant yet high sensitivity is noticed for each investigated defect depth as time elapses.

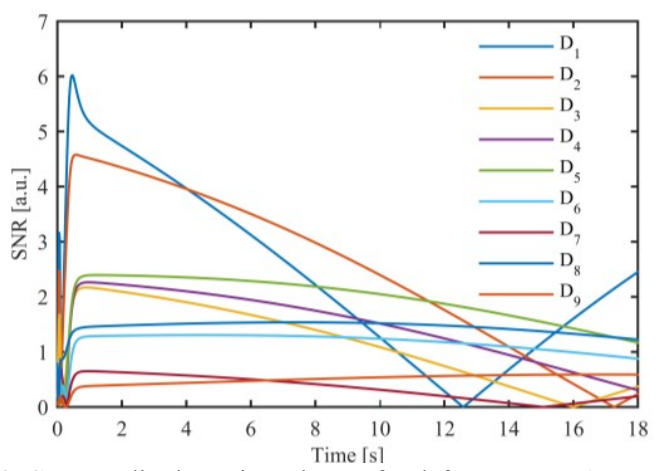

Fig. 8. SNR amplitude as time elapses for defects $\mathrm{D}_{1}-\mathrm{D}_{9}$, ARX model $(2,4,0)$. 


\section{FURTHER RESULTS}

The high fidelity of the $h[k]$ retrieved with ARX $(40,40,0)$ allows advanced post-processing algorithms to be faithfully implemented. In particular, the so-called "Time-Phase" algorithm has been here exploited [5], which is based on the application of Hilbert's Transform (HT) over the $h[k]$ to obtain $h[k]$ 's analytical signal. The block diagram in Fig.10 shows the Time-Phase algorithm applied over a $h[k]$ of a single $x-y$ pixel of the thermogram.

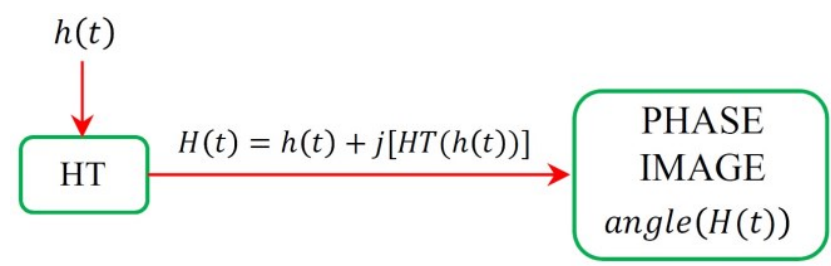

Fig.10. Block diagram of the Time-Phase algorithm. HT stands for Hilbert Transform, Re and $I M$ for real and imaginary part of the signal respectively.

The advantage of Time-Phase algorithm is that of producing images that are robust against local variation of sample emissivity and inhomogeneous illumination (as for any phase imaging algorithm), while allowing a time analysis to be still performed at the same time. Fig.11 depicts a series of thermograms obtained by applying the Time-Phase algorithm over the $h[k]$ 's retrieved with ARMA $(40,40,0)$ as time elapses. An enhanced detection capability for deeper defects $\left(\mathrm{D}_{7}-\mathrm{D}_{8}\right)$ is appreciated.

\section{CONCLUSIONS}

RPT was conducted over a CFRP benchmark containing artificial defects buried at different depths by means of an MLS modulated heating stimulus. An ARX $(40,40,0)$ model was proposed to characterize the system response and its reliability was demonstrated. The capability of the proposed model to extract the feature of interest, here signature of artificial defect into a CFRP sample, was investigated introducing an SNR merit parameter. Results show that the model is capable not only to detect defects IR signatures, but it is sensitive to different depths of the defects. Moreover, it has been shown that the selection of an ARX model having a lower order still represents a good choice in terms of defect detection capability but hampers a faithful defect characterization. Finally, it has been shown that the fidelity of the impulse response obtained with $\operatorname{ARX}(40,40,0)$ allows the Time-Phase algorithm to be fruitfully exploited for enhancing the detection capability of defects at deeper depths. At the best of our knowledge, this paper shows the first application of Time-Phase algorithm in RPT. Future works will investigate the robustness of the proposed ARX model on RPT test, whereas MLS having different timefrequency characteristics will be employed. A comparison with PuCT will be included as well.

\section{REFERENCES}

[1] X. Maldague, "Applications of Infrared Thermography”, Trends Opt. Nondestruct. Test., 2000, pp. 591-609.

[2] X. Maldague, F. Galmiche, A. Ziadi, "Advances in pulsed phase thermography”, Infrared physics \& technology, 2002, vol.43(3-5): pp. $175-181$.

[3] K. Chatterjee, S. Tuli, S. G. Pickering, D. P. Almond, "A comparison of the pulsed, lock-in and frequency modulated thermography nondestructive evaluation techniques", NDT \& E International , 2011, vol.44(7), pp. 655-667.

[4] S. Tuli, R. Mulavesaala, "Defect detection by pulse compression in frequency modulated thermal wave imaging", Quantitative InfraRed Thermography Journal, 2005, vol.2(1), pp. 41-54.

[5] N. Tabatabaei, A. Mandelis, B.T. Amaechi, "Thermophotonic radar imaging: An emissivity-normalized modality with advantages over phase lock-in thermography", Applied Physics Letters, 2011, vol.98(16): 163706.

[6] G. Silipigni, et al. "Optimization of the pulse-compression technique applied to the infrared thermography nondestructive evaluation", NDT \& E International, 2017, vol.87, pp. 100-110.

[7] S. Laureti, et al., "The use of pulse-compression thermography for detecting defects in paintings," NDT \& E International, 2018, vol.98, pp. 147-154.

[8] S. Laureti, et al. "Comparative study between linear and non-linear frequency-modulated pulse-compression thermography.", Applied Optics, 2018, 57.18: D32-D39.

[9] G. Dua, R. Mulaveesala, "Applications of Barker coded infrared imaging method for characterisation of glass fibre reinforced plastic materials," Electronics Letters, 2013, vol.49(17), pp. 1071-1073.

[10] J. C. Candoré, J.L. Bodnar, V. Detalle, P. Grossel, "Non-destructive testing of works of art by stimulated infrared thermography", The European Physical Journal Applied Physics, 2012, vol.57(2): 21002.

[11] B. R. Pramod, S. C. Bose, "System identification using ARMA modeling and neural networks", Journal of Engineering for Industry, 1993, vol.115(4), pp. 487-491.

[12] G. D'Angelo, S. Rampone, "Feature extraction and soft computing methods for aerospace structure defect classification," Measurement, 2016, vol.85, pp.192-209.

[13] K. J. Åström, P. Eykhoff, "System identification-a survey", Automatica, 1971, vol.7(2), pp. 123-162.
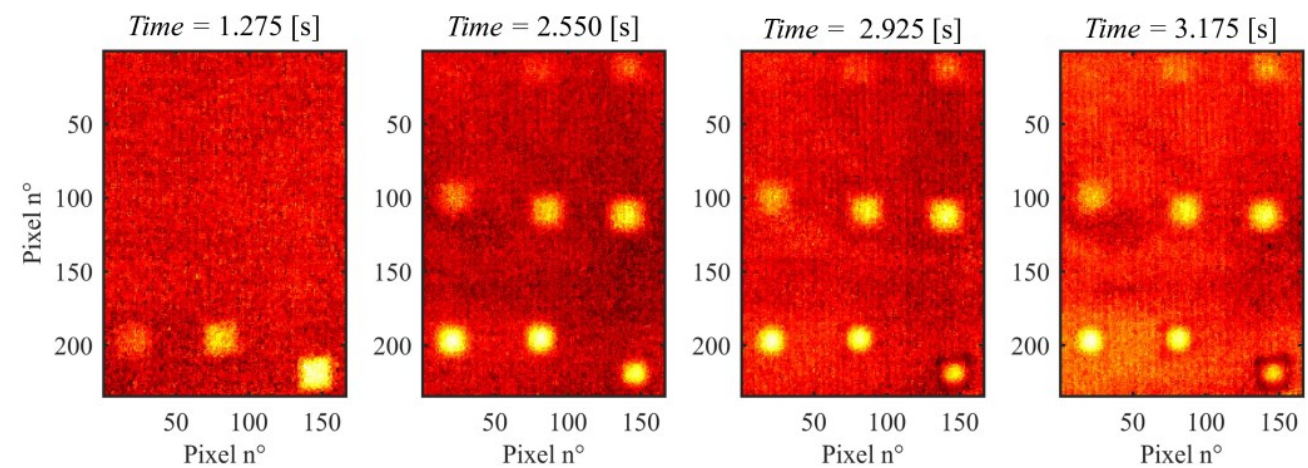

Fig.11. A series thermograms obtained by applying the Time-Phase algorithm over the h(t)'s retrieved with ARMA (40,40,0) as time elapses. 
[14] Y. Lu, F. Gao, "A novel time-domain auto-regressive model for structural damage diagnosis", Journal of Sound and Vibration, 2005, 283.3-5: pp. 1031-1049.

[15] S. M. Pandit, S. M. Wu, "Time Series and System Analysis with Applications", John Wiley \& Sons, Florida,1993.

[16] R. Diversi, R. Guidorzi, U. Soverini, "Identification of ARX and ARARX models in the presence of input and output noises.", European Journal of Control, 2010, vol.16(3), pp. 242-255.

[17] L. Ljung, "System identification", Signal analysis and prediction. Birkhäuser, Boston, MA, 1998, pp. 163-173.

[18] K. J. Åström, P. Eykhoff, "System identification-a survey", Automatica, 1971, vol.7(2), pp. 123-162.

[19] M. H Perrott, R. J. Cohen, "An efficient approach to ARMA modeling of biological systems with multiple inputs and delays", IEEE Transactions on Biomedical Engineering, 1996, vol.43(1), 1.

[20] S.W. Golomb."Shift register sequences", Aegean Park Press, 1982.

[21] D. Hutchins, P. Burrascano, L. Davis, S. Laureti, M. Ricci, "Coded waveforms for optimised air-coupled ultrasonic nondestructive evaluation", Ultrasonics, 2014, vol. 54(7), pp.1745-1759. 